\title{
COEFFICIENT ESTIMATES ASSOCIATED WITH A NEW SUBCLASS OF BI-UNIVALENT FUNCTIONS
}

\author{
A. Akgül, Ş. Altinkaya
}

ABstraCt. In our present investigation, we aim at introducing a new subclass of the function class $\Sigma$ of bi-univalent functions defined in the open unit disc $\mathbb{U}$. Furthermore, we establish bounds for the coefficients for this subclass and several related classes are also considered and connections to earlier known results are made.

2010 Mathematics Subject Classification: 30C45.

Keywords: Bi-univalent functions, bi-starlike functions, coefficient estimates.

\section{Introduction AND DeFinitions}

Let $\mathcal{A}$ indicate the class of functions $f$ which are analytic in the open unit disc $\mathbb{U}=\{z: z \in \mathbb{C}$ and $|z|<1\}$, of the form

$$
f(z)=z+\sum_{n=2}^{\infty} a_{n} z^{n} .
$$

Let $\mathcal{S}$ be the subclass of $A$ consisting of the form (1) which are univalent in $U$. It is well known that every function $f \in \mathcal{S}$ has an inverse $f^{-1}$, satisfying $f^{-1}(f(z))=$ $z,(z \in \mathbb{U})$ and $f\left(f^{-1}(w)\right)=w,\left(|w|<r_{0}(f), r_{0}(f) \geq \frac{1}{4}\right)$, where

$$
f^{-1}(w)=w-a_{2} w^{2}+\left(2 a_{2}^{2}-a_{3}\right) w^{3}-\left(5 a_{2}^{3}-5 a_{2} a_{3}+a_{4}\right) w^{4}+\cdots .
$$

A function $f \in \mathcal{A}$ is said to be bi-univalent in $\mathbb{U}$ if both $f$ and $f^{-1}$ are univalent in $\mathbb{U}$. Let $\Sigma$ denote the class of bi-univalent functions defined in the unit disc $\mathbb{U}$. For a brief history and interesting examples of functions in the class $\Sigma$, see the pioneering work on this area by Srivastava et al. [12], which has apparently revived the study of bi-univalent functions in recent years.

The research into $\Sigma$ was started by Lewin [10]. It focused on problems connected with coefficients and obtained the bound 1.51 for the modulus of the second coefficient $\left|a_{2}\right|$. Subsequently, Brannan and Clunie [6] conjectured that $\left|a_{2}\right| \leqq \sqrt{2}$ for 
$f \in \Sigma$. Later on, Netanyahu [11] showed that $\max \left|a_{2}\right|=\frac{4}{3}$ if $f(z) \in \Sigma$. Brannan and Taha [5] introduced certain subclasses of the bi-univalent function class $\Sigma$ similar to the familiar subclasses $\mathcal{S}^{\star}(\beta)$ and $\mathcal{K}(\beta)$ of starlike and convex functions of order $\beta$ $(0 \leqq \beta<1)$ in $\mathbb{U}$, respectively (see $[11])$. The classes $\mathcal{S}_{\Sigma}^{\star}(\beta)$ and $\mathcal{K}_{\Sigma}(\beta)$ of bi-starlike functions of order $\beta$ in $\mathbb{U}$ and bi-convex functions of order $\beta$ in $\mathbb{U}$, corresponding to the function classes $\mathcal{S}^{\star}(\beta)$ and $\mathcal{K}(\beta)$, were also introduced analogously. For each of the function classes $\mathcal{S}_{\Sigma}^{\star}(\beta)$ and $\mathcal{K}_{\Sigma}(\beta)$, they found non-sharp estimates for the initial coefficients. Recently, motivated substantially by the aforementioned work on this area Srivastava et al. [12], many authors investigated the coefficient bounds for various subclasses of bi-univalent functions (see, for example, [2], [7], [13]). Not much is known about the bounds on the general coefficient $\left|a_{n}\right|$ for $n \geqq 4$. In the literature, there are only a few works determining the general coefficient bounds for $\left|a_{n}\right|$ for the analytic bi-univalent functions (see, for example, [4], [8], [9]). The coefficient estimate problem for each of the coefficients $\left|a_{n}\right|(n \in \mathbb{N} \backslash\{1,2\} ; \mathbb{N}=\{1,2,3, \cdots\})$ is still an open problem.

In our present investigation, we aim at introducing a new subclass of the function class $\Sigma$ of bi-univalent functions defined in the open unit disc $\mathbb{U}$. Furthermore, we find estimates on the coefficients $\left|a_{2}\right|$ and $\left|a_{3}\right|$ for functions in this new subclass of the function class $\Sigma$ employing the techniques used earlier by Altınkaya and Yalçın [2] (see also [1]).

We note the following definition required for obtaining our results.

Definition 1. Let the functions $h, p: \mathbb{U} \rightarrow \mathbb{C}$ be so constrained that

$$
\min \{\Re(h(z)), \Re(p(z))\}>0
$$

and

$$
h(0)=p(0)=1 .
$$

\section{Coefficient Estimates for the Function Class $S_{\Sigma}^{h, p}(\alpha)$}

We begin this section by introducing the function class $S_{\Sigma}^{h, p}(\alpha)$ and finding the estimates on the coefficients $\left|a_{2}\right|$ and $\left|a_{3}\right|$ for functions in this class.

Definition 2. A function $f \in \Sigma$ is said to be in the class $S_{\Sigma}^{h, p}(\alpha), 0<\alpha \leq 1$, if the following conditions are satisfied:

$$
\frac{1}{2}\left(\frac{z f^{\prime}(z)}{f(z)}+\left(\frac{z f^{\prime}(z)}{f(z)}\right)^{\frac{1}{\alpha}}\right) \in h(\mathbb{U}) \quad(z \in \mathbb{U})
$$


and

$$
\frac{1}{2}\left(\frac{w g^{\prime}(w)}{g(w)}+\left(\frac{w g^{\prime}(w)}{g(w)}\right)^{\frac{1}{\alpha}}\right) \in p(\mathbb{U}) \quad(w \in \mathbb{U})
$$

where $g(w)=f^{-1}(w)$.

Remark 1. There are many choices of $h, p$ and $\alpha$ which would provide interesting subclasses of class $S_{\Sigma}^{h, p}(\alpha)$. For example,

1. For $0<\alpha \leq 1$ and $h(z)=p(z)=\left(\frac{1+z}{1-z}\right)^{\lambda}$ where $(0<\lambda \leq 1)$ it can be directly verified that the functions $h(z)$ and $p(z)$ satisfy the hypotheses of Definition 1. Now if $f \in S_{\Sigma}(\lambda, \alpha)$ then

$$
f \in \Sigma, \quad\left|\arg \frac{1}{2}\left(\frac{z f^{\prime}(z)}{f(z)}+\left(\frac{z f^{\prime}(z)}{f(z)}\right)^{\frac{1}{\alpha}}\right)\right|<\frac{\lambda \pi}{2} \quad(0<\lambda \leq 1, \quad z \in U)
$$

and

$$
\left|\arg \frac{1}{2}\left(\frac{w g^{\prime}(w)}{g(w)}+\left(\frac{w g^{\prime}(w)}{g(w)}\right)^{\frac{1}{\alpha}}\right)\right|<\frac{\lambda \pi}{2} \quad(0<\lambda \leq 1, \quad w \in U) .
$$

Therefore in this case, the class $S_{\Sigma}^{h, p}(\alpha)$ reduces to class $S_{\Sigma}(\lambda, \alpha)$ which is defined by Altınkaya and Yalçın [3].

2. For $0<\alpha \leq 1$ and $h(z)=p(z)=\frac{1+(1-2 \beta) z}{1-z}$ where $(0 \leq \beta<1)$ it can be directly verified that the functions $h(z)$ and $p(z)$ satisfy the hypotheses of Definition 1. Now if $f \in S_{\Sigma}(\lambda, \beta)$ then

$$
f \in \Sigma, \quad \Re\left(\frac{1}{2}\left(\frac{z f^{\prime}(z)}{f(z)}+\left(\frac{z f^{\prime}(z)}{f(z)}\right)^{\frac{1}{\alpha}}\right)\right)>\beta \quad(0 \leq \beta<1,0<\alpha \leq 1, z \in U)
$$

and

$$
\Re\left(\frac{1}{2}\left(\frac{w g^{\prime}(w)}{g(w)}+\left(\frac{w g^{\prime}(w)}{g(w)}\right)^{\frac{1}{\alpha}}\right)\right)>\beta \quad(0 \leq \beta<1,0<\alpha \leq 1, w \in U) .
$$

Therefore in this case, the class $S_{\Sigma}^{h, p}(\alpha)$ reduces to class $S_{\Sigma}(\lambda, \beta)$ which is defined by Altınkaya and Yalçın [3].

3. For $\alpha=1$ and $h(z)=p(z)=\left(\frac{1+z}{1-z}\right)^{\lambda}$ where $(0<\lambda \leq 1)$ it can be directly verified that the functions $h(z)$ and $p(z)$ satisfy the hypotheses of Definition 1. Now if $f \in S_{\Sigma}(\alpha)$ then

$$
f \in \Sigma, \quad\left|\arg \left(\frac{z f^{\prime}(z)}{f(z)}\right)\right|<\frac{\lambda \pi}{2} \quad(0<\lambda \leq 1, \quad z \in U)
$$


and

$$
\left|\arg \left(\frac{w g^{\prime}(w)}{g(w)}\right)\right|<\frac{\lambda \pi}{2} \quad(0<\lambda \leq 1, \quad w \in U) .
$$

Therefore in this case, the class $S_{\Sigma}^{h, p}(\alpha)$ reduces to class $S_{\Sigma}(\alpha)$ which is defined by Brannan and Taha [5] (see also [14]).

4. For $\alpha=1$ and $h(z)=p(z)=\frac{1+(1-2 \beta) z}{1-z}$ where $(0 \leq \beta<1)$ it can be directly verified that the functions $h(z)$ and $p(z)$ satisfy the hypotheses of Definition 1. Now if $f \in S_{\Sigma}(\beta)$ then

$$
f \in \Sigma, \quad \Re\left(\frac{z f^{\prime}(z)}{f(z)}\right)>\beta \quad(0 \leq \beta<1,0<\alpha \leq 1, z \in U)
$$

and

$$
\Re\left(\frac{w g^{\prime}(w)}{g(w)}\right)>\beta \quad(0 \leq \beta<1,0<\alpha \leq 1, w \in U) .
$$

Therefore in this case, the class $S_{\Sigma}^{h, p}(\alpha)$ reduces to class $S_{\Sigma}(\beta)$ which is defined by Brannan and Taha [5] (see also [14]).

Theorem 1. Let $f$ given by (1) be in the class $S_{\Sigma}^{h, p}(\alpha)$. Then

$$
\left|a_{2}\right| \leq \min \left\{\sqrt{\frac{2\left(\left|h^{\prime}(0)\right|^{2}+\left|p^{\prime}(0)\right|^{2}\right) \alpha^{2}}{(1+\alpha)^{2}}}, \sqrt{\frac{\left(\left|h^{\prime \prime}(0)\right|+\left|p^{\prime \prime}(0)\right|\right) \alpha^{2}}{2 \alpha^{2}+\alpha+1}}\right\}
$$

and

$$
\left|a_{3}\right| \leq \min \left\{\begin{array}{l}
\frac{2\left(\left|h^{\prime}(0)\right|^{2}+\left|p^{\prime}(0)\right|^{2}\right) \alpha}{(1+\alpha)^{2}}+\frac{\left(\left|h^{\prime \prime}(0)\right|+\left|p^{\prime \prime}(0)\right|\right) \alpha}{4(1+\alpha)}, \\
\frac{\left(6 \alpha^{3}+5 \alpha^{2}+\alpha\right)\left|h^{\prime \prime}(0)\right|}{4(1+\alpha)\left(2 \alpha^{2}+\alpha+1\right)}+\frac{\left(2 \alpha^{3}+3 \alpha^{2}-\alpha\right)\left|p^{\prime \prime}(0)\right|}{4(1+\alpha)\left(2 \alpha^{2}+\alpha+1\right)}
\end{array} .\right.
$$

Proof. Let $f \in S_{\Sigma}^{h, p}(\alpha)$. It follows from (2) and (3) that

$$
\frac{1}{2}\left(\frac{z f^{\prime}(z)}{f(z)}+\left(\frac{z f^{\prime}(z)}{f(z)}\right)^{\frac{1}{\alpha}}\right)=h(z)
$$

and

$$
\frac{1}{2}\left(\frac{w g^{\prime}(w)}{g(w)}+\left(\frac{w g^{\prime}(w)}{g(w)}\right)^{\frac{1}{\alpha}}\right)=p(w),
$$

where $h(z)$ and $p(w)$ satisfy the conditions of Definition 1. Furthermore, the functions $h(z)$ and $p(w)$ have the following Taylor-Maclaurin series expansions:

$$
h(z)=1+h_{1} z+h_{2} z^{2}+\cdots
$$


and

$$
p(w)=1+p_{1} w+p_{2} w^{2}+\cdots,
$$

respectively. Thus, upon comparing the corresponding coefficients in (6) and (7), we get

$$
\begin{gathered}
\frac{\alpha+1}{2 \alpha} a_{2}=h_{1}, \\
\frac{\alpha+1}{2 \alpha}\left(2 a_{3}-a_{2}^{2}\right)+\frac{1-\alpha}{4 \alpha^{2}} a_{2}^{2}=h_{2},
\end{gathered}
$$

and

$$
\begin{gathered}
-\frac{\alpha+1}{2 \alpha} a_{2}=p_{1}, \\
\frac{\alpha+1}{2 \alpha}\left(3 a_{2}^{2}-2 a_{3}\right)+\frac{1-\alpha}{4 \alpha^{2}} a_{2}^{2}=p_{2} .
\end{gathered}
$$

From (8) and (10) we obtain

$$
h_{1}=-p_{1}
$$

and

$$
\frac{(\alpha+1)^{2}}{2 \alpha^{2}} a_{2}^{2}=h_{1}^{2}+p_{1}^{2} .
$$

Now, by adding (9) to (11), we find that

$$
\frac{2 \alpha^{2}+\alpha+1}{2 \alpha^{2}} a_{2}^{2}=h_{2}+p_{2}
$$

which gives us the desired estimate on $\left|a_{2}\right|$ as asserted in (4).

Next, in order to find the bound on $\left|a_{3}\right|$, by subtracting (11) from (9), we obtain

$$
\frac{2(\alpha+1)}{\alpha}\left(a_{3}-a_{2}^{2}\right)=h_{2}-p_{2} .
$$

Therefore, in view of (12) and (13), we have

$$
a_{3}=\frac{2\left(h_{1}^{2}+p_{1}^{2}\right) \alpha}{(\alpha+1)^{2}}+\frac{\left(h_{2}-p_{2}\right) \alpha}{2(\alpha+1)}
$$

and

$$
a_{3}=\frac{2\left(h_{2}+p_{2}\right) \alpha^{2}}{2 \alpha^{2}+\alpha+1}+\frac{\left(h_{2}-p_{2}\right) \alpha}{2(\alpha+1)} .
$$

which completes the proof of Theorem 1 . 


\section{Corollaries and Consequences}

Corollary 2. If we let

$$
h(z)=p(z)=\left(\frac{1+z}{1-z}\right)^{\lambda}=1+2 \lambda z+2 \lambda^{2} z^{2}+\ldots \quad(0<\lambda \leq 1),
$$

then inequalities (4) and (5) become

$$
\left|a_{2}\right| \leq \min \left\{\frac{4 \alpha \lambda}{1+\alpha}, 2 \lambda \alpha \sqrt{\frac{2}{2 \alpha^{2}+\alpha+1}}\right\}=2 \lambda \alpha \sqrt{\frac{2}{2 \alpha^{2}+\alpha+1}}
$$

and

$$
\left|a_{3}\right| \leq \min \left\{\frac{16 \lambda^{2} \alpha^{2}}{(1+\alpha)^{2}}+\frac{2 \lambda^{2} \alpha}{1+\alpha}, \frac{8 \alpha^{2} \lambda^{2}}{2 \alpha^{2}+\alpha+1}\right\} .
$$

Corollary 3. If we let

$h(z)=p(z)=\frac{1+(1-2 \beta) z}{1-z}=1+2(1-\beta) z+2(1-\beta) z^{2}+\cdots \quad(0 \leq \beta<1)$, then inequalities (4) and (5) become

$$
\left|a_{2}\right| \leq \min \left\{\frac{4 \alpha(1-\beta)}{1+\alpha}, 2 \alpha \sqrt{\frac{2(1-\beta)}{2 \alpha^{2}+\alpha+1}}\right\} .
$$

and

$$
\left|a_{3}\right| \leq \min \left\{\frac{16(1-\beta)^{2} \alpha^{2}}{(1+\alpha)^{2}}+\frac{2(1-\beta) \alpha}{1+\alpha}, \frac{8 \alpha^{2}(1-\beta)}{2 \alpha^{2}+\alpha+1}\right\} .
$$

Taking $\alpha=1$ in Theorem 1 , we get

Corollary 4. If $f \in S_{\Sigma}^{h, p}$ then

$$
\left|a_{2}\right| \leq \min \left\{\sqrt{\frac{\left|h^{\prime}(0)\right|^{2}+\left|p^{\prime}(0)\right|^{2}}{2}}, \sqrt{\frac{\left|h^{\prime \prime}(0)\right|+\left|p^{\prime \prime}(0)\right|}{4}}\right\}
$$

and

$$
\left|a_{3}\right| \leq \min \left\{\frac{\left|h^{\prime}(0)\right|^{2}+\left|p^{\prime}(0)\right|^{2}}{2}+\frac{\left|h^{\prime \prime}(0)\right|+\left|p^{\prime \prime}(0)\right|}{8}, \frac{3\left|h^{\prime \prime}(0)\right|}{8}+\frac{\left|p^{\prime \prime}(0)\right|}{8}\right\}
$$

Corollary 5. If we let

$$
h(z)=p(z)=\left(\frac{1+z}{1-z}\right)^{\lambda}=1+2 \lambda z+2 \lambda^{2} z^{2}+\ldots \quad(0<\lambda \leq 1)
$$


then inequalities (15) and (16) become

$$
\left|a_{2}\right| \leq \min \{2 \lambda, \sqrt{2} \lambda\}=\sqrt{2} \lambda
$$

and

$$
\left|a_{3}\right| \leq \min \left\{5 \lambda^{2}, 2 \lambda^{2}\right\}=2 \lambda^{2} .
$$

Remark 2. Corollary 8 provides an improvement estimates obtained by Altinkaya and Yalçın [3].

Corollary 6. (see [3]) If we let

$$
h(z)=p(z)=\frac{1+(1-2 \beta) z}{1-z}=1+2(1-\beta) z+2(1-\beta) z^{2}+\cdots \quad(0 \leq \beta<1),
$$

then inequalities (15) and (16) become

$$
\left|a_{2}\right| \leq \min \{2(1-\beta), \sqrt{2(1-\beta)}\}=\sqrt{2(1-\beta)}
$$

and

$$
\left|a_{3}\right| \leq \min \left\{4(1-\beta)^{2}+(1-\beta), 2(1-\beta)\right\}=4(1-\beta)^{2}+(1-\beta) .
$$

\section{Acknowledgements.}

The authors are extremely grateful to the reviewers for a careful reading of the manuscript and making valuable suggestions leading to a better presentation of the paper.

\section{REFERENCES}

[1] Ş. Altınkaya, S. Yalçın, Coefficient estimates for two new subclasses of Biunivalent functions, Acta Universitatis Apulensis 43 (2015), 53-63.

[2] Ş. Altınkaya, S. Yalçın, Coefficient estimates for two new subclasses of Biunivalent functions with respect to symmetric points, Journal of Function Spaces, Article ID 145242, 2015 (2015), 5 pages.

[3] Ş. Altınkaya, S. Yalçın, Coefficient bounds for certain subclasses of bi-univalent functions, Creat. Math. Inform. 24 (2015), 101-106.

[4] S.. Altınkaya, S. Yalçın, Faber polynomial coefficient bounds for a subclass of bi-univalent functions, C. R. Acad. Sci. Paris Ser. I 353 (2015), 1075-1080. 
A. Akgül, Ş. Altmkkaya - Coefficient estimates ...

[5] D. A. Brannan, T. S. Taha, On some classes of bi-univalent functions, in: S.M. Mazhar, A. Hamoui, N.S. Faour (Eds.), Math. Anal. and Appl., Kuwait; February 18-21, 1985, in: KFAS Proceedings Series, vol. 3, Pergamon Press, Elsevier Science Limited, Oxford, 1988, pp. 53-60. see also Studia Univ. Babeş-Bolyai Math. 31 (1986), 70-77.

[6] D. A. Brannan, J. G. Clunie, Aspects of comtemporary complex analysis, (Proceedings of the NATO Advanced Study Instute Held at University of Durham:July 1-20, 1979), New York: Academic Press, (1980).

[7] B. A. Frasin, M. K. Aouf, New subclasses of bi-univalent functions, Applied Mathematics Letters 24 (2011), 1569-1573.

[8] S. G. Hamidi, J. M. Jahangiri, Faber polynomial coefficient estimates for analytic bi-close-to-convex functions, C. R. Acad. Sci. Paris Ser. I 352 (2014), 17-20.

[9] J. M. Jahangiri, S. G. Hamidi, Coefficient estimates for certain classes of biunivalent functions, Int. J. Math. Math. Sci. ArticleID 190560, 2013 (2013), 4 pages.

[10] M. Lewin, On a coefficient problem for bi-univalent functions, Proceeding of the American Mathematical Society 18 (1967), 63-68.

[11] E. Netanyahu, The minimal distance of the image boundary from the origin and the second coefficient of a univalent function in $|z|<1$, Archive for Rational Mechanics and Analysis 32 (1969), 100-112.

[12] H. M. Srivastava, A. K. Mishra, P. Gochhayat, Certain subclasses of analytic and bi-univalent functions, Applied Mathematics Letters 23 (2010), 1188-1192.

[13] H. M. Srivastava, S. B. Joshi, S. S. Joshi, H. Pawar, Coefficient estimates for certain subclasses of meromorphically bi-univalent functions, Palest. J. Math. 5 (Special Issue: 1) (2016), 250-258.

[14] T. S. Taha, Topics in univalent function theory, Ph.D. Thesis, University of London, (1981).

Arzu Akgül

Department of Mathematics, Faculty of Arts and Science, Kocaeli University,

Kocaeli, Turkey

email: akgul@kocaeli.edu.tr

Şahsene Altınkaya

Department of Mathematics, Faculty of Arts and Science,

Uludag University,

Bursa, Turkey

email: sahsene@uludag.edu.tr 